\title{
The effect of solar activity on the Doppler and multipath spread of HF signals received over paths oriented along the midlatitude trough
}

\author{
A. J. Stocker ${ }^{1}$ and E. M. Warrington ${ }^{1}$ \\ Received 19 July 2010; revised 16 November 2010; accepted 9 December 2010; published 11 February 2011.
}

[1] Measurements of the Doppler and delay spread associated with HF signals propagating along an oblique $(1440 \mathrm{~km})$ path tangential to the midlatitude ionospheric trough are presented for sunspot maximum and minimum. During the day, Doppler spread is independent of solar activity, but for winter and equinoctial nights, it is very much higher at sunspot maximum. The delay spread is also generally higher at sunspot maximum for all seasons and times of day. For sunspot minimum, measurements from a second, longer path $(1800 \mathrm{~km})$ are also presented. The observed Doppler and delay spreads are similar for both paths. Finally, a novel method of more accurately deriving the delay spread defined by the International Telecommunication Union (i.e., the largest delay spread including all modes that have a peak power within a user-defined threshold of that of the strongest mode) from Voice of America coverage analysis program (VOACAP) predictions is presented. For the first time, the predicted values are compared with the measured delay spreads and, while there is generally good agreement at sunspot minimum, the agreement at sunspot maximum tends to be poor because the behavior of the high-order ionospheric modes (e.g., 3F2) is not well predicted by VOACAP.

Citation: Stocker, A. J., and E. M. Warrington (2011), The effect of solar activity on the Doppler and multipath spread of HF signals received over paths oriented along the midlatitude trough, Radio Sci., 46, RS1014, doi:10.1029/2010RS004482.

\section{Introduction}

[2] The midlatitude or subauroral trough is a region of the ionosphere of a few degrees in latitudinal extent where the electron density (and hence the critical frequency and the maximum usable frequency) is reduced [Rodger et al., 1992]. The trough generally occurs at night during the winter and equinoctial months, and rarely during the summer. The reduction in electron density means that the signal radiated by $\mathrm{HF}$ radio communication systems operating under the trough will no longer be ionospherically reflected along the great circle path. However, the signal can still be reflected from gradients in the poleward and equatorward walls of the trough or scattered from irregularities embedded in the poleward wall or in the auroral region which lies just poleward of the trough.

\footnotetext{
${ }^{1}$ Department of Engineering, University of Leicester, Leicester, UK.

Copyright 2011 by the American Geophysical Union. 0048-6604/11/2010RS004482
}

If these signals then reach the receiver, they will arrive from directions away from the great circle and at times delayed with respect to normal propagation. Observations and simulations of these effects have been reported in a number of papers [Siddle et al., 2004a, 2004b; Stocker et al., 2007, 2009; Zaalov et al., 2005, 2007]. As well as directional and time of flight effects, the channel characteristics of such signals can be significantly different from normal, on great circle propagation. For example, scattering from irregularities will tend to increase the Doppler spread of the signal, while if the signal arrives over several different paths (perhaps some on and some off-great circle) this will increase the delay spread. The experimentally observed delay and Doppler spreads on a path affected by the trough at solar maximum were presented by Warrington and Stocker [2003].

[3] The data rate and robustness of radio communication systems in a large part depend on channel characteristics such as the delay and Doppler spread and therefore these ionospheric effects can have a significant effect on the data rates achieved. For example, when Digital Radio Mondiale (DRM) is operated at $21 \mathrm{kbit} / \mathrm{s}$ it can tolerate a delay spread of $5 \mathrm{~ms}$ and a Doppler spread of $3 \mathrm{~Hz}$ 


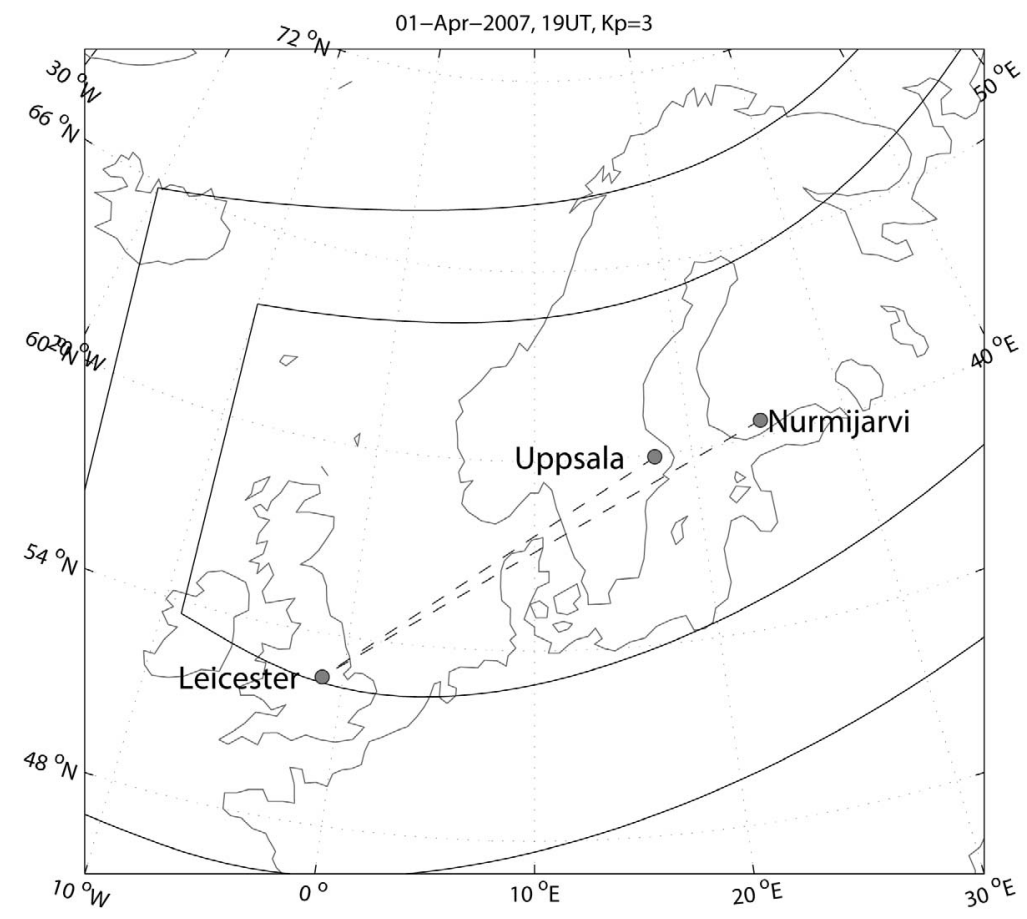

Figure 1. A map showing the location of the transmitter sites (Uppsala and Nurmijärvi) and the receiver site (Leicester). The location of the trough predicted by the Halcrow and Nisbet [1977] model for 1 April 2007, at 1900 UT and for Kp $=3$ is also presented.

(provided SNR $>21 \mathrm{~dB}$ ). However, reducing the data rate to $7.6 \mathrm{kbit} / \mathrm{s}$ increases the delay and Doppler spread tolerances to $7 \mathrm{~ms}$ and $6 \mathrm{~Hz}$, respectively, provided the SNR is greater than $17 \mathrm{~dB}$ [Smith and Angling, 2003]. The performance of other modem types (e.g., those that conform to the STANAG 4285 or MIL-STD-188-110B standards) is also constrained by the delay and Doppler spread channel characteristics (see Warrington and Stocker [2003] for a table of delay and Doppler spread tolerances for a variety of modem types and data rates).

[4] In this paper, we present experimental observations of delay and Doppler spreads together with predictions of delay spreads for three situations. Measurements are first presented for a path from Uppsala-Leicester during sunspot maximum, second for the same path, but at solar minimum, and lastly for a slightly longer path (Nurmijärvi-Leicester) also at solar minimum. The predictions of delay spread are based on the definition recently introduced by the International Telecommunication Union Radiocommunication Sector (ITU-R) [ITU-R, 2007; Barclay et al., 2009] although VOACAP [Lane, 2001] is used rather than ITU-R [2009]. The results given here form the first systematic comparison between predicted and experimental values of the new delay spread parameter.

\section{Experiment}

[5] The transmitter and receiver sites employed in the HF propagation experiments undertaken at sunspot maximum (2000-2002) and sunspot minimum (2006 to date) are presented in Figure 1. Both paths (Uppsala-Leicester and Nurmijärvi-Leicester, henceforth abbreviated as UL and NL, respectively) lie approximately parallel to the midlatitude trough (also shown in Figure 1) and within about five degrees of azimuth (as observed at Leicester) of each other. Although the NL path is longer $(1800 \mathrm{~km})$ than UL $(1440 \mathrm{~km})$, some similarities in ionospheric propagation are expected, e.g., 1-hop propagation via both $E$ and $F$ regions is possible on either path. The method used to transmit and collect the observations has been discussed in detail elsewhere [e.g., Siddle et al., 2004a; Stocker et al., 2009]. However, in brief, a $100 \mathrm{~W}$, 13 bit Barker encoded BPSK signal (1667 baud in 2001 and 2000 baud in 2006 onward) was radiated from the two transmitter sites on a range of frequencies in the lower half of the HF band (4.6-18.4 MHz) and received 
Table 1. Experimental Parameters of the Three Sets of Measurements

\begin{tabular}{cccc}
\hline & Data Included & $\begin{array}{c}\text { Sample } \\
\text { Resolution } \\
\text { (min) }\end{array}$ & $\begin{array}{c}\text { Doppler } \\
\text { Resolution } \\
\text { (Hz) }\end{array}$ \\
\hline $\begin{array}{c}\text { Uppsala-Leicester } \\
\text { (UL01) Sunspot } \\
\text { maximum }\end{array}$ & $\begin{array}{c}\text { January 2001 to } \\
\text { December 2001 }\end{array}$ & 3 & 0.625 \\
$\begin{array}{c}\text { Uppsala-Leicester } \\
\text { (UL06) Sunspot }\end{array}$ & $\begin{array}{c}\text { August 2006 to } \\
\text { January 2008 }\end{array}$ & 2 & 0.5 \\
$\begin{array}{c}\text { minimum } \\
\text { Nurmijärvi-Leicester } \\
\text { (NL06) Sunspot } \\
\text { minimum }\end{array}$ & $\begin{array}{c}\text { December 2006 to } \\
\text { April 2010 }\end{array}$ & 2 & 0.5 \\
\hline
\end{tabular}

${ }^{\mathrm{a}}$ Time of flight measurements from August to December 2006 are unreliable, so delay spreads from this period are not included in the results presented below.

on a circularly disposed antenna array at a field site $15 \mathrm{~km}$ to the south of Leicester. The parameters that can be measured by the system include time of flight (TOF, and hence delay spread), Doppler (and hence Doppler spread), signal-to-noise ratio, and, although not discussed in this paper, directional characteristics (i.e., azimuth and elevation). There were some slight operational differences between the systems used at sunspot maximum and minimum and these are summarized in Table 1. The Doppler resolution presented here is determined from the length of the data sample (e.g., 2 s in 2006 onward), with the error from phase noise being considerably below this. Given the unusually long period of sunspot minimum in the current cycle, there is a strong justification in including all of the NL data from December 2006 to April 2010 as representing sunspot minimum.

[6] In order to compare the observations presented here with earlier experiments [Davies and Cannon, 1993; Warrington and Stocker, 2003], the multipath and Doppler spread parameters are defined here in a similar manner to that employed by Angling et al. [1998]. Two multipath (or delay) spread parameters were employed, since one, effective multipath spread, gave better results than the composite multipath spread when employed in modem tests using an HF channel simulator [Angling and Davies, 1999]. However, for this paper a new multipath spread parameter has been employed based on that given by ITU-R [2007]. This parameter can be derived for both observations and from prediction codes (such as Voice of America coverage analysis program, VOACAP [Lane, 2001]), so it provides an opportunity to compare measurements with predictions.

[7] The four spread parameters are defined as follows:

[8] The composite multipath spread (CMPS) is the time between the rising edge of the first detected mode and the falling edge of the last detected mode, with a correction applied for the width of the transmitted pulse. Weak modes, i.e., those with an integrated power (i.e., summed over all TOF where the mode exists) of more than $12 \mathrm{~dB}$ below the integrated power of the strongest mode, were excluded from the processing.

[9] The effective multipath spread (EMPS) is calculated for a pair of modes from the multipath separation between the rising edge of the central $80 \%$ power region of the first (in delay time) mode and the trailing edge of the central $80 \%$ power region of the last mode (corrected for the transmitted pulse width). The separation is then weighted by the ratio of the two modes' total powers ensuring that the ratio is always equal or less than unity. This procedure is applied to each pair of modes in turn and the effective multipath spread taken as the maximum value found. If only a single mode is present, then the effective multipath spread is taken to be the mode's $80 \%$ power spread, again corrected for the width of the transmitted pulse.

[10] The composite Doppler spread is the narrowest spectral width containing $80 \%$ of the received signal power. In all cases, a correction is applied for the base noise level.

[11] The ITU-R multipath spread (IMPS), as stated above, is based on the definition presented by $I T U-R$ [2007] and is calculated by finding the largest delay spread including all modes which have a peak power within $10 \mathrm{~dB}$ of that of the strongest mode. Unlike the other delay-spread parameters (i.e., CMPS and EMPS), IMPS can be derived for both observations and for predictions such as those made by VOACAP (note that VOACAP is used instead of ITU-R [2009] because running VOACAP using 'method 25, all mode tables' gives easy access to the SNR and time delays of each individual ionospheric mode). Although using the median SNR values from VOACAP to determine which modes are within the $10 \mathrm{~dB}$ window provides a quick calculation of the delay spread [e.g., Rogers, 2003], this does not make use of the SNR probability distribution function that VOACAP provides through the inclusion of upper and lower decile values. Following Lane [2001], the median and upper and lower decile values of SNR in a $1 \mathrm{~Hz}$ bandwidth, $S N R_{50}, S N R_{90}$, and $S N R_{10}$, respectively (note the change in notation from Lane) are given by:

$$
\begin{gathered}
S N R_{50}=P_{50}-N_{50} \\
S N R_{90}=S N R_{50}+\sqrt{d P_{90}^{2}+d N_{90}^{2}} \\
S N R_{10}=S N R_{50}-\sqrt{d P_{10}^{2}+d N_{10}^{2}}
\end{gathered}
$$



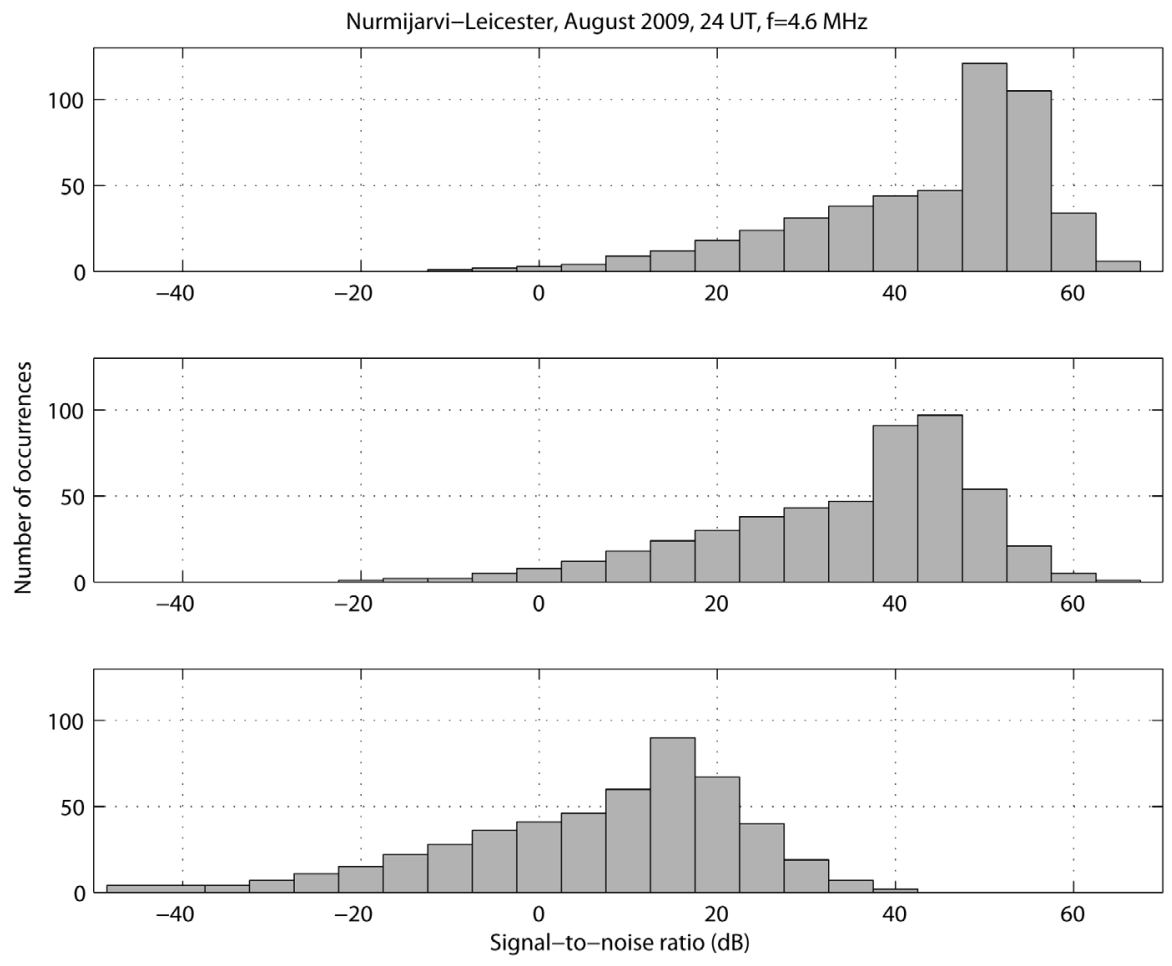

Figure 2. VOACAP predictions of SNR distributions for a signal of $4.6 \mathrm{MHz}$ propagating on the Nurmijärvi-Leicester path in August 2009 at 2400 UT. The modes are (top) $1 F 2$ (TOF $=6.38 \mathrm{ms,}$ $S N R_{50}=49.2 \mathrm{~dB}, S N R_{10}=22.7 \mathrm{~dB}, S N R_{90}=56.8 \mathrm{~dB}$ ), (middle) low-angle $2 \mathrm{~F} 2$ (TOF $=7.41 \mathrm{~ms}$, $\left.S N R_{50}=39.6 \mathrm{~dB}, S N R_{10}=13.0 \mathrm{~dB}, S N R_{90}=49.9 \mathrm{~dB}\right)$, and (bottom) high-angle $2 \mathrm{~F} 2$ (TOF = $\left.8.35 \mathrm{~ms}, S N R_{50}=11.2 \mathrm{~dB}, S N R_{10}=-15.3 \mathrm{~dB}, S N R_{90}=24.4 \mathrm{~dB}\right)$. The $1 \mathrm{E}$ and $2 \mathrm{E}$ modes predicted for this time are omitted since they are very weak $\left(S N R_{50}<-200 \mathrm{~dB}\right)$.

where, $P_{50}$ is the median signal power, $N_{50}$ is the median noise power, $d P_{90}=\mathrm{P}_{90}-\mathrm{P}_{50}$ and $d P_{10}=P_{50}-P_{10}$, with $P_{10}$ and $P_{90}$ being the lower and upper decile signal powers, respectively, and $d N_{90}=\mathrm{N}_{90}-N_{50}$ and $d N_{10}=$ $N_{50}-N_{10}$ with $N_{10}$ and $N_{90}$ being the lower and upper decile noise powers (in a $1 \mathrm{~Hz}$ bandwidth), respectively. All powers are given in $\mathrm{dB}$. If it is assumed that the SNR probability distribution function is lognormal in form, then the resultant Gaussian distributions will have standard deviations of approximately $\left(\mathrm{SNR}_{90}-\mathrm{SNR}_{50}\right) / 1.28$ and $\left(\mathrm{SNR}_{50}-\mathrm{SNR}_{10}\right) / 1.28$ on the upper and lower sides of the distribution, respectively. In order to use this information to calculate the delay spread, a brute-force method was adopted such that:

[12] 1. The SNR distribution is determined with sample SNR values calculated at a fixed interval in probability. An example of the resulting distributions is given in Figure 2. Several points can be noted: each mode has a longer tail for SNR values below the peak and, in this example, the distributions of the three modes overlap in SNR.
[13] 2. All possible combinations of SNR values are then taken in turn and in each case, the strongest mode and those modes that have an SNR within $10 \mathrm{~dB}$ of the strongest mode are found. The IMPS is then determined from the delays of each of the modes within the $10 \mathrm{~dB}$ SNR window. This procedure assumes that the SNR values of each mode are independent, which is a good assumption if the reflection points of the modes are sufficiently separated in distance. For a given interval in probability, this is the rate-determining step since the number of calculations (and the time taken) goes roughly as the power of the number of modes. To ensure this step executes in a reasonable time, the interval of probability is varied with the number of modes, e.g., in Figure 2, with three modes, the interval in probability is $0.2 \%$ (i.e., 500 samples define the distribution) resulting in an execution time of $3 \mathrm{~min}$ (on a $2.4 \mathrm{GHz}$ processor). For five modes, although the interval is increased to $2.5 \%$ (and hence the number of samples representing the distribution is reduced to 40) the execution time is increased to $15 \mathrm{~min}$. If more than five modes are present, only the five 
Table 2. Percentage Occurrence of IMPS Derived From VOACAP Predictions for Signal of 4.6 MHz Propagating on the Nurmijärvi-Leicester Path in August 2009 at 2400 UT

\begin{tabular}{cc}
\hline IMPS $(\mathrm{ms})$ & Occurrence $(\%)$ \\
\hline 0.00 & 61.68 \\
0.94 & 0.72 \\
1.03 & 34.93 \\
1.97 & 2.67 \\
\hline
\end{tabular}

strongest (in terms of median SNR) are included in the calculation. Tests on a significant number of cases for the paths under investigation have shown that reducing the step size further and allowing more than five modes tends to have only a minimal effect on the results produced for considerable increases in execution time. The execution times given above are large relative to the time taken to run VOACAP, and this means that the current method is not really suitable for a practical prediction system However, a Monte Carlo approach will be adopted to provide faster access to IMPS predictions; this will be the subject of a future paper.

[14] 3. Once all SNR states have been found in step 2 above, the probability of occurrence of each value of IMPS is determined and the mean and median calculated. For the example given in Figure 2, the probabilities are given in Table 2. The spreads in Table 2 correspond to the single-moded case (i.e., no other mode is within $10 \mathrm{~dB}$ of the peak) at $0 \mathrm{~ms}$, the high- and low-angle $2 \mathrm{~F} 2$ modes being in the SNR window (0.94 ms), the 1F2 and lowangle $2 \mathrm{~F} 2(1.03 \mathrm{~ms})$, and the $1 \mathrm{~F} 2$ and high-angle $2 \mathrm{~F} 2$ modes $(1.97 \mathrm{~ms})$. For this example, simply taking the median SNR values results in an IMPS of $1.03 \mathrm{~ms}$, while properly including the SNR probability distribution functions results in a median IMPS of $0 \mathrm{~ms}$ and a mean IMPS of $0.42 \mathrm{~ms}$ (with a standard deviation of $0.55 \mathrm{~ms}$ ). For the paths considered, this reduction in IMPS when the SNR distributions are included is typical. For example, for $8 \mathrm{MHz}$ on the NL06 path, the simple method overestimates the mean IMPS by $0.2 \mathrm{~ms}$ in $25 \%$ of cases, and by $0.86 \mathrm{~ms}$ in $10 \%$ of cases, with a worst case of $1.2 \mathrm{~ms}$.

[15] For consistency with previous results [Angling et al., 1998; Warrington and Stocker, 2003], only observations with a SNR (in a $3 \mathrm{kHz}$ bandwidth) of at least $6 \mathrm{~dB}$ were included in the statistical analysis (cases affected by interferers were removed manually). Winter is defined as November-January, spring as February-April, summer as May-July, and autumn as August-October. Daytime is considered as the $6 \mathrm{~h}$ period centered on noon (UT) and nighttime as the $6 \mathrm{~h}$ period centered on midnight (UT). Note that since the publication of Warrington and Stocker [2003], the 2001 data have been reprocessed making three changes. First, the way the power profile with delay time was determined was changed, second weak modes were removed from the calculation of CMPS (see above), and third a small error in the calculation of the Doppler spread was corrected. This error led to an underestimation of the Doppler spread on the rare occasions when there was more than one peak in the Doppler spectrum. The change to the power profile calculation (projecting the data in the delay-Doppler into the delay axis, rather than, effectively, summing it) has led to an increase in the apparent SNR and a greater sensitivity of the various delay spread parameters to the presence of weaker ionospheric modes (particularly nighttime sporadic E). Removal of the weak modes from the calculation of CMPS has decreased the influence of noise on the derived value.

\section{Observations}

[16] The medians of the observed cumulative distributions of the spread parameters are not included in this paper for reasons of space, although the general behavior of the medians for Uppsala-Leicester, 2001 (UL01, see Table 1 for the other abbreviations used for various paths and years) can be found in the work of Warrington and Stocker [2003]. The changes in the calculation method described above have had little effect on the medians of Doppler spread (changes are $0.6 \mathrm{~Hz}$ or less) and EMPS (changes are $0.1 \mathrm{~ms}$ or less), and where the original CMPS was less than $0.6 \mathrm{~ms}$ (changes are $0.1 \mathrm{~ms}$ or less). However, where the original value of CMPS was greater than $0.6 \mathrm{~ms}$, the new value is approximately a third of the original value. For the observations taken since 2006, the median Doppler spread was $0.5 \mathrm{~Hz}$ for all situations, except for the UL path for summer night (4.64 MHz), winter day and night $(6.95 \mathrm{MHz})$, and winter day $(8.01 \mathrm{MHz})$ where it was $1.0 \mathrm{~Hz}$. The median EMPS was $0.1-0.2 \mathrm{~ms}$ for all cases, while the median CMPS was also $0.1-0.2 \mathrm{~ms}$ for all cases except for the UL path for equinox day at $6.95 \mathrm{MHz}$ where it was $0.4 \mathrm{~ms}$.

\subsection{Behavior of Spreads at Sunspot Maximum and Minimum}

[17] The 95th percentiles of the various spreads for different seasons and times of day are given in Tables 3-8. Note that a change in the frequency set employed in 2001 and $2006(18.4 \mathrm{MHz}$ in 2001 was replaced by $8.01 \mathrm{MHz}$ in 2006) means that no observations for $8.01 \mathrm{MHz}$ are available in 2001 and those for $18.4 \mathrm{MHz}$ have been omitted. Examination of the results for UL01 and UL06 in Tables 3-8 reveals that, given the difference in Doppler frequency resolution, there tends to be no significant difference in the daytime Doppler spreads between sunspot maximum and sunspot minimum. The exception to this is winter daytime where the Doppler spread for the higher frequencies is higher, at $2.5 \mathrm{~Hz}$, at sunspot maximum compared to $1.5 \mathrm{~Hz}$ at sunspot minimum. However, during winter and equinox nights, the Doppler 
Table 3. Spreads at the 95th Percentile During Winter Daytime (0900-1500 UT, November-January) for UppsalaLeicester 2001 and 2006-2007 and Nurmijärvi-Leicester $2006-2010^{\mathrm{a}}$

\begin{tabular}{|c|c|c|c|c|c|c|}
\hline & \multicolumn{6}{|c|}{ Frequency $(\mathrm{MHz})$} \\
\hline & 4.64 & 6.95 & 8.01 & 10.39 & 11.12 & 14.36 \\
\hline \multicolumn{7}{|c|}{ Number of Samples } \\
\hline UL01 & 106 & 3,569 & - & 6,657 & 6,468 & 5,755 \\
\hline UL06 & 43 & 7,581 & 16,569 & 14,826 & 15,984 & 4,004 \\
\hline NL06 & 4 & 862 & 461 & 5,667 & 17,145 & 27,120 \\
\hline \multicolumn{7}{|c|}{ Doppler $(\mathrm{Hz})$} \\
\hline UL01 & 1.9 & 1.9 & - & 2.5 & 2.5 & 2.5 \\
\hline UL06 & 1.5 & 1.5 & 1.5 & 1.5 & 1.5 & 1.5 \\
\hline NL06 & - & 1.5 & 1.5 & 1.5 & 1.5 & 1.5 \\
\hline \multicolumn{7}{|c|}{ Multipath (ms) } \\
\hline UL01 & 2.8 & 2.4 & - & 2.2 & 2.4 & 1.3 \\
\hline UL06 & 2.0 & 1.6 & 1.1 & 0.2 & 0.2 & 0.2 \\
\hline NL06 & - & 0.8 & 0.7 & 0.5 & 0.3 & 0.2 \\
\hline \multicolumn{7}{|c|}{ Effective Multipath (ms) } \\
\hline UL01 & 1.3 & 1.2 & - & 1.2 & 1.4 & 0.8 \\
\hline UL06 & 1.2 & 0.9 & 0.8 & 0.2 & 0.2 & 0.2 \\
\hline NL06 & - & 0.4 & 0.5 & 0.5 & 0.2 & 0.2 \\
\hline
\end{tabular}

${ }^{\mathrm{a} U L 01, ~ U p p s a l a-L e i c e s t e r ~ 2001 ; ~ U L 06, ~ U p p s a l a-L e i c e s t e r ~ 2006-~}$ 2007; NL06, Nurmijärvi-Leicester 2006-2010.

spread is considerably higher at sunspot maximum than at sunspot minimum for all frequencies except 4.64 MHz. For the EMPS, the difference between sunspot maximum and minimum is clear with the higher values occurring at sunspot maximum, with the differences being particularly marked at the higher frequencies. Furthermore,

Table 4. As Table 3, Except Spreads at the 95th Percentile During Winter Nighttime (2100-0300 UT, November-January)

\begin{tabular}{|c|c|c|c|c|c|c|}
\hline & \multicolumn{6}{|c|}{ Frequency $(\mathrm{MHz})$} \\
\hline & 4.64 & 6.95 & 8.01 & 10.39 & 11.12 & 14.36 \\
\hline \multicolumn{7}{|c|}{ Number of Samples } \\
\hline UL01 & 3,394 & 2,330 & - & 1,201 & 983 & 112 \\
\hline UL06 & 1,027 & 281 & 1,374 & 366 & 253 & 40 \\
\hline NL06 & 431 & 1,294 & 321 & 41 & 8 & 16 \\
\hline \multicolumn{7}{|c|}{ Doppler $(\mathrm{Hz})$} \\
\hline UL01 & 1.9 & 4.4 & - & 12.0 & 13.3 & 10.7 \\
\hline UL06 & 1.5 & 1.5 & 1.5 & 1.5 & 1.5 & 1.5 \\
\hline NL06 & 1.5 & 1.5 & 1.5 & 1.5 & - & 1.5 \\
\hline \multicolumn{7}{|c|}{ Multipath ( $\mathrm{ms}$ ) } \\
\hline UL01 & 2.2 & 2.2 & - & 3.5 & 3.6 & 1.5 \\
\hline UL06 & 0.3 & 0.2 & 0.1 & 0.1 & 0.1 & 0.1 \\
\hline NL06 & 0.3 & 0.2 & 0.2 & 0.1 & - & 0.1 \\
\hline \multicolumn{7}{|c|}{ Effective Multipath (ms) } \\
\hline UL01 & 1.0 & 0.8 & - & 1.3 & 1.4 & 0.6 \\
\hline UL06 & 0.2 & 0.2 & 0.1 & 0.1 & 0.1 & 0.1 \\
\hline NL06 & 0.3 & 0.2 & 0.2 & 0.1 & - & 0.1 \\
\hline
\end{tabular}

Table 5. As Table 3, Except Spreads at the 95th Percentile During Equinox Daytime (0900-1500 UT, February-April and August-October)

\begin{tabular}{|c|c|c|c|c|c|c|}
\hline & \multicolumn{6}{|c|}{ Frequency $(\mathrm{MHz})$} \\
\hline & 4.64 & 6.95 & 8.01 & 10.39 & 11.12 & 14.36 \\
\hline \multicolumn{7}{|c|}{ Number of Samples } \\
\hline UL01 & 51 & 6,028 & - & 15,704 & 15,432 & 13,785 \\
\hline UL06 & 4 & 7,294 & 17,777 & 22,227 & 20,123 & 4,356 \\
\hline NL06 & 0 & 273 & 957 & 7,987 & 25,980 & 35,558 \\
\hline \multicolumn{7}{|c|}{ Doppler $(\mathrm{Hz})$} \\
\hline UL01 & 1.9 & 1.9 & - & 1.9 & 1.9 & 1.9 \\
\hline UL06 & - & 1.5 & 1.5 & 1.5 & 1.5 & 1.5 \\
\hline NL06 & - & 1.5 & 1.5 & 1.5 & 1.5 & 1.5 \\
\hline \multicolumn{7}{|c|}{ Multipath (ms) } \\
\hline UL01 & 1.2 & 2.5 & - & 2.5 & 2.7 & 1.3 \\
\hline UL06 & - & 2.1 & 1.4 & 0.3 & 0.3 & 0.1 \\
\hline NL06 & - & 1.3 & 0.9 & 0.5 & 0.5 & 0.2 \\
\hline \multicolumn{7}{|c|}{ Effective Multipath (ms) } \\
\hline UL01 & 0.3 & 1.2 & - & 1.3 & 1.3 & 0.7 \\
\hline UL06 & - & 1.2 & 0.9 & 0.3 & 0.3 & 0.1 \\
\hline NL06 & - & 0.6 & 0.7 & 0.5 & 0.4 & 0.2 \\
\hline
\end{tabular}

while at sunspot minimum there is a clear tendency for the EMPS to decrease with increasing frequency for all seasons and times, during sunspot maximum there are some exceptions to this (e.g., winter day and night, and equinox and summer day). The behavior of CMPS at sunspot maximum and minimum is similar to that of EMPS, i.e., CMPS is higher during sunspot maximum than at sunspot minimum. While clearly there is a difference

Table 6. As Table 3, Except Spreads at the 95th Percentile During Equinox Nighttime (2100-0300 UT, February-April and August-October)

\begin{tabular}{lllllll}
\hline & \multicolumn{7}{c}{ Frequency (MHz) } \\
\cline { 2 - 7 } & 4.64 & 6.95 & 8.01 & 10.39 & 11.12 & 14.36 \\
\hline UL01 & 6,509 & 9,927 & - & 4,138 & 2,671 & 203 \\
UL06 & 915 & 1,345 & 2,892 & 793 & 595 & 131 \\
NL06 & 543 & 1,131 & 560 & 568 & 652 & 9 \\
& \multicolumn{7}{c}{ Number of Samples } \\
UL01 & 1.9 & 2.5 & - & 5.7 & 6.3 & 5.1 \\
UL06 & 1.5 & 1.5 & 1.5 & 1.5 & 1.5 & 1.5 \\
NL06 & 1.5 & 1.5 & 1.5 & 1.5 & 1.5 & - \\
& \multicolumn{7}{c}{ Doppler $(\mathrm{Hz})$} \\
UL01 & 3.1 & 2.0 & - & 0.8 & 0.7 & 0.3 \\
UL06 & 1.1 & 0.3 & 0.2 & 0.1 & 0.1 & 0.1 \\
NL06 & 0.4 & 0.2 & 0.2 & 0.2 & 0.2 & - \\
& \multicolumn{7}{c}{ Multipath (ms) } \\
UL01 & 1.3 & 0.8 & - & 0.6 & & \\
UL06 & 0.4 & 0.3 & 0.2 & 0.1 & 0.1 & 0.1 \\
NL06 & 0.2 & 0.2 & 0.2 & 0.2 & 0.2 & - \\
\hline
\end{tabular}


Table 7. As Table 3, Except Spreads at the 95th Percentile During Summer Daytime (0900-1500 UT, May-July)

\begin{tabular}{|c|c|c|c|c|c|c|}
\hline & \multicolumn{6}{|c|}{ Frequency $(\mathrm{MHz})$} \\
\hline & 4.64 & 6.95 & 8.01 & 10.39 & 11.12 & 14.36 \\
\hline \multicolumn{7}{|c|}{ Number of Samples } \\
\hline UL01 & 0 & 2,608 & - & 9,353 & 9,177 & 8,134 \\
\hline UL06 & 0 & 436 & 4,997 & 5,222 & 5,538 & 1,715 \\
\hline NL06 & 0 & 8 & 5 & 683 & 2,470 & 6,421 \\
\hline \multicolumn{7}{|c|}{ Doppler $(\mathrm{Hz})$} \\
\hline UL01 & - & 1.3 & - & 1.9 & 1.9 & 1.9 \\
\hline UL06 & - & 1.5 & 1.5 & 1.5 & 1.5 & 1.5 \\
\hline NL06 & - & - & - & 1.5 & 1.5 & 1.5 \\
\hline \multicolumn{7}{|c|}{ Multipath (ms) } \\
\hline UL01 & - & 2.1 & - & 2.2 & 1.9 & 0.5 \\
\hline UL06 & - & 1.9 & 1.1 & 0.4 & 0.3 & 0.1 \\
\hline NL06 & - & - & - & 0.3 & 0.2 & 0.2 \\
\hline \multicolumn{7}{|c|}{ Effective Multipath (ms) } \\
\hline UL01 & - & 0.6 & - & 1.1 & 0.9 & 0.3 \\
\hline UL06 & - & 1.1 & 0.8 & 0.4 & 0.3 & 0.1 \\
\hline NL06 & - & - & - & 0.3 & 0.2 & 0.2 \\
\hline
\end{tabular}

between the results obtained at sunspot maximum and sunspot minimum, it is interesting to note that there is no systematic change in spread with smoothed sunspot number (SSN) over the period 2006-2010 (SSN varies from 16 in August 2006, to 4 in July 2009, to a predicted value of 11 in February 2010).

[18] The values in Tables 3-8 can be compared with those suggested for use in testing HF modems with ionospheric channel simulators in the work of ITU-R [2000]. The observed median Doppler spread is $0.5 \mathrm{~Hz}$, which corresponds to the representative value for midlatitudes under moderate conditions in the work of $I T U-R$ [2000]. However, the 95th percentile has values between 1.5 and $2.5 \mathrm{~Hz}$ for sunspot maximum day and summer night and for all cases at sunspot minimum, and these are higher than the representative values for midlatitudes and lower than those for high latitudes (except for quiet conditions). During winter and equinoctial nights at sunspot maximum, the 95th percentile of Doppler spread is up to $13 \mathrm{~Hz}$, a value that is similar to that given by $I T U-R$ [2000] for high latitudes under moderate conditions. The observed median values of CMPS $(0.1-0.2 \mathrm{~ms})$ are smaller than the values given by $I T U-R$ [2000] for midlatitude quiet conditions. While the measured 95 th percentile values are strongly dependent on frequency, season, and sunspot value, in general they lie between the values suggested for moderate conditions at midlatitudes $(1 \mathrm{~ms})$ and high latitudes (3 ms).

\subsection{The Effect of Path Length on Spreads}

[19] The difference in path length between UL and NL (approximately $400 \mathrm{~km}$ ) results in no difference in
Doppler spread. In general, there is little difference in EMPS except at the lowest frequencies where the EMPS is higher for the shorter path during the day and for summer nights, and at higher frequencies during equinoxes (for both day and night) where the opposite is the case (i.e., the EMPS is slightly higher for the longer path). Similar behavior is found for the CMPS. The behavior at the lower frequencies arises because the ionospheric modes (e.g., 1F2, 2F2, etc.) will be closer together in TOF for the longer path leading to lower spreads. While it is difficult to draw any firm conclusions about the behavior of the higher frequencies since the difference in spread is generally at the limit of the precision of measurements, these values may represent occasions where a second ionospheric mode is present in the longer path but not in the shorter one.

\subsection{Observations and Predictions of IMPS}

[20] The upper decile values of IMPS predicted by VOACAP (version 10.0123 has been used throughout) for seasons and times of day as defined in Tables 3-8 are presented in Table 9. VOACAP has been run with input parameters that reproduce the experimental configuration as closely as possible, although the sporadic E model has been switched off. In order to allow the VOACAP predictions to be compared with the observations, the predicted IMPS probability distribution functions from the appropriate individual months and hours (i.e., similar to that given in Table 2) have been combined. The combined distribution has then been used to determine the upper decile value of IMPS. An example of a combined

Table 8. As Table 3, Except Spreads at the 95th Percentile During Summer Nighttime (2100-0300 UT, May-July)

\begin{tabular}{lllllll}
\hline & \multicolumn{7}{c}{ Frequency (MHz) } \\
\cline { 2 - 7 } & 4.64 & 6.95 & 8.01 & 10.39 & 11.12 & 14.36 \\
\hline UL01 & 2,924 & 8,288 & - & 7,186 & 5,868 & 1,378 \\
UL06 & 734 & 2,325 & 5,204 & 2,128 & 1,521 & 136 \\
NL06 & 115 & 970 & 352 & 1,498 & 3,325 & 748 \\
& \multicolumn{7}{c}{ Number of Samples } \\
UL01 & 1.3 & 1.9 & - & 1.9 & 1.9 & 1.9 \\
UL06 & 1.5 & 1.5 & 1.5 & 1.5 & 1.5 & 1.5 \\
NL06 & 1.5 & 1.5 & 1.5 & 1.5 & 1.5 & 1.5 \\
& \multicolumn{7}{c}{ Doppler $(H z)$} \\
UL01 & 2.8 & 3.0 & - & 0.8 & 0.6 & 0.2 \\
UL06 & 2.5 & 1.4 & 0.5 & 0.2 & 0.2 & 0.1 \\
NL06 & 1.3 & 0.5 & 0.2 & 0.2 & 0.2 & 0.2 \\
& \multicolumn{7}{c}{ Multipath (ms) } \\
UL01 & 1.3 & 1.3 & - & 0.4 & \\
UL06 & 1.3 & 0.8 & 0.4 & 0.2 & 0.2 & 0.1 \\
NL06 & 0.6 & 0.4 & 0.2 & 0.2 & 0.2 & 0.2 \\
\hline
\end{tabular}


Table 9. Observed and Predicted Values of the Upper Decile of ITU Multipath Spread (ms) at a Threshold of $-10 \mathrm{~dB}$

\begin{tabular}{|c|c|c|c|c|c|c|c|c|c|c|c|c|}
\hline & \multicolumn{2}{|c|}{4.64} & \multicolumn{2}{|c|}{6.95} & \multicolumn{2}{|c|}{8.01} & \multicolumn{2}{|c|}{10.39} & \multicolumn{2}{|c|}{11.12} & \multicolumn{2}{|c|}{14.36} \\
\hline & VOA & Observed & VOA & Observed & VOA & Observed & VOA & Observed & VOA & Observed & VOA & Observed \\
\hline \multicolumn{13}{|c|}{ Winter } \\
\hline \multicolumn{13}{|l|}{ Day } \\
\hline UL01 & 2.2 & 1.9 & 1.0 & 2.0 & - & - & 1.1 & 2.1 & 1.0 & 2.3 & 1.1 & 1.1 \\
\hline UL06 & 1.7 & 2.2 & 0.9 & 1.8 & 0.9 & 1.1 & 0.2 & 0.0 & 0.2 & 0.0 & 0.0 & 0.0 \\
\hline NL06 & 1.5 & - & 1.4 & 0.9 & 0.8 & 0.7 & 0.6 & 0.6 & 0.1 & 0.0 & 0.1 & 0.0 \\
\hline \multicolumn{13}{|l|}{ Night } \\
\hline UL01 & 1.7 & 1.8 & 0.1 & 1.7 & - & - & 0.0 & 2.8 & 0.0 & 3.1 & 0.0 & 0.8 \\
\hline UL06 & 0.0 & 0.0 & 0.0 & 0.0 & 0.0 & 0.0 & 0.0 & 0.0 & 0.0 & 0.0 & 0.0 & 0.0 \\
\hline NL06 & 0.0 & 0.0 & 0.0 & 0.0 & 0.0 & 0.0 & 0.0 & 0.0 & 0.0 & - & 0.0 & 0.0 \\
\hline \multicolumn{13}{|c|}{ Summer } \\
\hline \multicolumn{13}{|l|}{ Day } \\
\hline UL01 & 0.4 & - & 2.3 & 1.7 & - & - & 0.8 & 2.0 & 1.0 & 1.6 & 0.9 & 0.6 \\
\hline UL06 & 1.9 & - & 2.1 & 1.8 & 0.5 & 1.1 & 0.6 & 0.6 & 0.7 & 0.0 & 0.6 & 0.0 \\
\hline NL06 & 1.8 & - & 1.6 & - & 1.7 & - & 0.4 & 0.0 & 0.6 & 0.0 & 0.6 & 0.0 \\
\hline \multicolumn{13}{|l|}{ Night } \\
\hline UL01 & 1.7 & 2.5 & 1.6 & 2.6 & - & - & 0.0 & 0.7 & 0.0 & 0.7 & 0.0 & 0.0 \\
\hline UL06 & 1.5 & 2.8 & 0.1 & 1.3 & 0.0 & 0.6 & 0.0 & 0.0 & 0.0 & 0.0 & 0.0 & 0.0 \\
\hline NL06 & 1.2 & 1.2 & 0.0 & 0.6 & 0.1 & 0.0 & 0.0 & 0.0 & 0.0 & 0.0 & 0.0 & 0.0 \\
\hline \multicolumn{13}{|c|}{ Equinox } \\
\hline Day & & & & & & & & & & & & \\
\hline UL01 & 2.4 & 1.1 & 2.1 & 2.2 & - & - & 1.1 & 2.4 & 1.1 & 2.4 & 0.8 & 1.1 \\
\hline UL06 & 2.0 & - & 1.8 & 2.1 & 1.0 & 1.4 & 0.4 & 0.4 & 0.5 & 0.0 & 0.0 & 0.0 \\
\hline NL06 & 2.0 & - & 1.6 & 1.1 & 1.4 & 1.1 & 0.3 & 0.6 & 0.3 & 0.3 & 0.4 & 0.0 \\
\hline \multicolumn{13}{|l|}{ Night } \\
\hline UL01 & 1.7 & 2.6 & 0.0 & 1.6 & - & - & 0.0 & 0.8 & 0.0 & 0.8 & 0.0 & 0.0 \\
\hline UL06 & 0.0 & 0.7 & 0.0 & 0.0 & 0.0 & 0.0 & 0.0 & 0.0 & 0.0 & 0.0 & 0.0 & 0.0 \\
\hline NL06 & 0.0 & 0.0 & 0.0 & 0.0 & 0.0 & 0.0 & 0.0 & 0.0 & 0.0 & 0.0 & 0.0 & 0.0 \\
\hline
\end{tabular}

distribution is given in Figure 3 (top) (this is discussed below).

[21] Examination of Table 9 reveals that, as might be expected, the behavior of IMPS with frequency and solar activity generally follows that of EMPS and CMPS, i.e., the spread tends to be higher at solar maximum and to decrease with increasing frequency. At sunspot maximum, while agreement between the VOACAP predictions and observed values of IMPS is occasionally found (e.g., winter day at $4.64 \mathrm{MHz}$ and $14.36 \mathrm{MHz}$ ), there appear to be a large number of cases where the agreement is poor, broadly divided into three types as follows: (1) The predictions are less than the measured values for 10.39 and $11.12 \mathrm{MHz}$ during the day for all seasons (and for 6.95 $\mathrm{MHz}$ during winter day). (2) The predictions are less than the measured values for all frequencies for propagation at night (although for $14.36 \mathrm{MHz}$ this is only true during the winter). (3) The predictions are higher than the measured values for equinox day at $4.64 \mathrm{MHz}$ (and marginally for winter day).

[22] An example of the occurrence of the predicted and observed delay spreads for the first type of poor agreement (in this case winter day-equinox day is similar) is given in Figure 3. The predicted and measured delay spreads arising from the various combinations of ionospheric modes are in good agreement, e.g., the spreads from 0.6 to $1.0 \mathrm{~ms}$ associated with the $1 \mathrm{~F} 2$ and $2 \mathrm{~F} 2$ modes. However, there is a significant difference in the relative occurrence of the various modes, e.g., the $3 \mathrm{~F} 2$ and 2F2 modes are far stronger in the measurements than the predictions and, therefore contribute to the IMPS more often and the $0 \mathrm{~ms}$ spread associated with singlemoded propagation is less common in the observations. Note that the IMPS arising from the combination of the $1 \mathrm{E}$ and 1F2 modes is missing from the measurements because the separation in delay of these two modes is less than the transmitted pulse width (0.6 ms in 2001). In the summer day, the difference between the observed and predicted IMPS arises from a $2 \mathrm{~F} 2$ mode that is observed (with a TOF that varies considerably from hour to hour and from day to day) but not predicted by VOACAP for either 10.39 or $11.12 \mathrm{MHz}$.

[23] For winter and equinox nights, the second type of poor agreement described above arises from the presence of propagation modes that arrive from off-great circle with long TOF, the signal having generally been scattered from irregularities embedded in the poleward wall of the trough or the auroral oval (e.g., see Siddle et al. [2004b] for details). On many occasions, there is a sporadic $\mathrm{E}$ mode present, and this combined with the off-great circle 

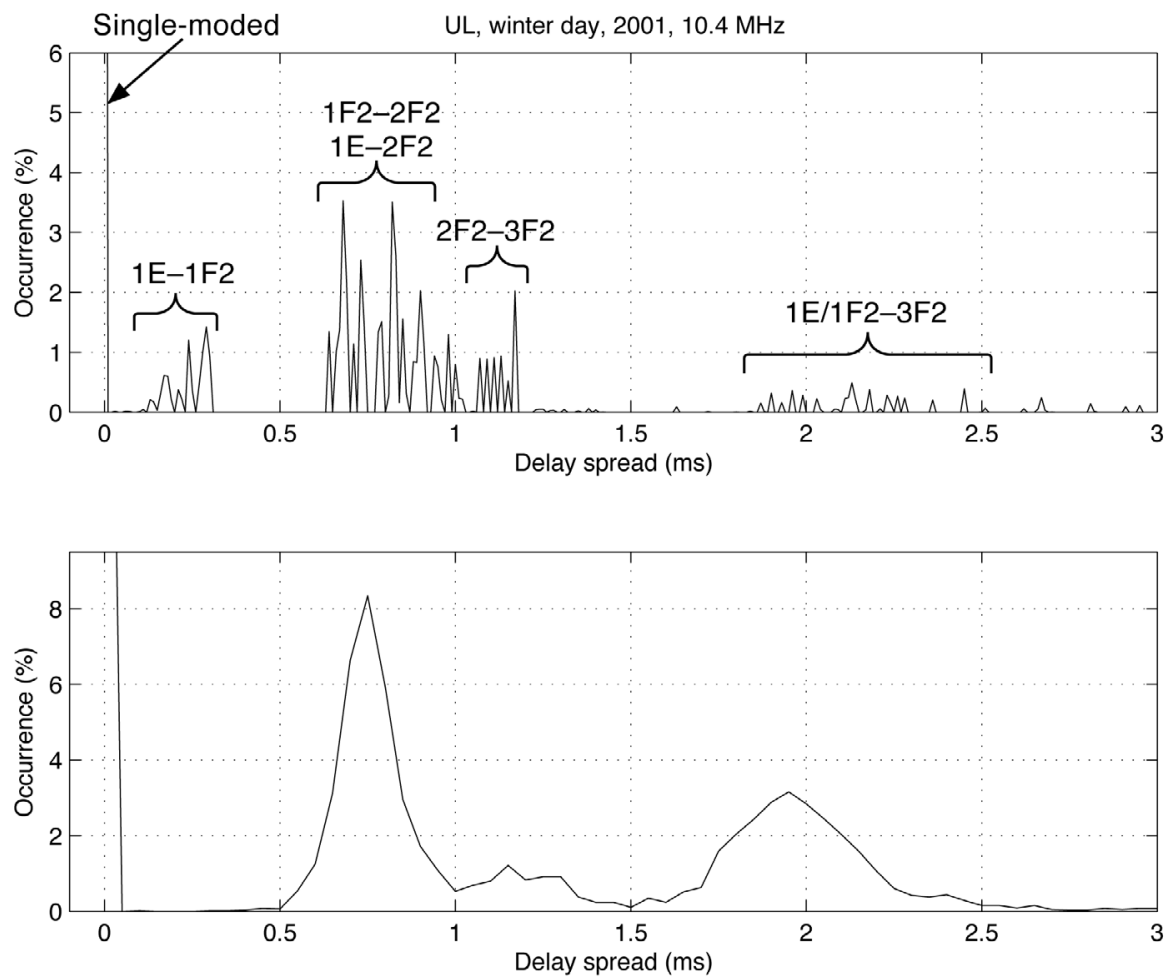

Figure 3. The probability of occurrence of IMPS for UL01, winter day. (top) The VOACAP predictions (with the ionospheric modes responsible for producing the spread indicated) and (bottom) the observed values. Note that the single-moded value (close to $0 \mathrm{~ms}$ in Figures 3 (top) and 3 (bottom)) has been cut off in order to emphasize the occurrence at higher spreads (the single-moded occurrence is $44 \%$ and $31 \%$ for Figures 3 (top) and 3 (bottom), respectively).

mode results in a high value of IMPS. VOACAP is not able to predict either the off-great circle propagation or (given the input parameters used) the sporadic E mode, and hence returns an IMPS of $0 \mathrm{~ms}$. For summer nights, the cause of the discrepancy between prediction and experiment depends on the frequency. For 10.39 and $11.12 \mathrm{MHz}$, it is the presence of sporadic $\mathrm{E}$, when combined with the observed (and predicted) 1F2 mode, which leads to a high value of IMPS. For the lower frequencies (i.e., 4.64 and $6.95 \mathrm{MHz}$ ), the observed TOF for the $2 \mathrm{~F} 2$ mode is sometimes higher than that predicted by VOACAP and a 3F2 mode is occasionally present in the observations which is either not predicted by VOACAP (6.95 MHz) or is predicted to have a low SNR (4.64 MHz). For example, for 01 UT in July, VOACAP predicts a TOF of $6.96 \mathrm{~ms}$ for the $2 \mathrm{~F} 2$ mode, while the observed value is often as high as $8 \mathrm{~ms}$ together with a $3 \mathrm{~F} 2$ mode in the range $8-10 \mathrm{~ms}$.

[24] In the third type of poor agreement, for equinox day the predicted IMPS is greater than that observed for the lowest frequency $(4.64 \mathrm{MHz})$. However, note that there are relatively few observations here (51, see Table 5) because the strong $D$ region absorption around noon usually reduces the signal to relatively low levels. For most of the cases, the observed propagation is via a $1 \mathrm{E}$ or $2 \mathrm{E}$ mode with what are likely to be $1 \mathrm{~F} 2$ and $2 \mathrm{~F} 2$ modes occurring on only one day (8 September 2001). The VOACAP predictions vary considerably across the months included (i.e., February-April and AugustOctober), although the presence of a variety of $\mathrm{E}$ modes (1E, 2E, and $3 \mathrm{E}$ all tend to be strong modes) is fairly fixed, the $2 \mathrm{~F} 2,3 \mathrm{~F} 2$, and $3 \mathrm{~F} 1$ modes tend to occur in different combinations for different months and times. The existence of both $E$ region and high-order $F$ region modes leads to the high value of predicted IMPS.

[25] At sunspot minimum, there is generally a good agreement between the VOACAP predictions of IMPS and the observed values for both paths, with summer nights for the lowest two frequencies and winter day for $6.95 \mathrm{MHz}$ on the UL06 path being notable exceptions. In each case, the observed spread is higher because of the presence of modes not predicted by VOACAP, i.e., for 
summer nights at $6.95 \mathrm{MHz}$, it is the existence of a $2 \mathrm{~F} 2$ mode in the observations, for $4.64 \mathrm{MHz}$, the occurrence of 3F2 and 4F2 modes, and for winter day at $6.95 \mathrm{MHz}$ the presence of a $3 \mathrm{~F} 2$ mode.

\section{Concluding Remarks}

[26] Observations (and in the case of IMPS, predictions) of delay and Doppler spreads in an HF channel have been presented for conditions of sunspot minimum and sunspot maximum. The spreads observed at sunspot minimum on two, almost parallel, paths of different lengths $(1440 \mathrm{~km}$ and $1800 \mathrm{~km})$ are also compared. The main results can be summarized as follows:

[27] 1. Except for winter, the daytime Doppler spreads are similar at sunspot maximum and sunspot minimum. In winter, the Doppler spread is slightly higher during sunspot maximum.

[28] 2. For winter and equinoctial nights, the Doppler spread is significantly higher during sunspot maximum. This occurs when the signal arrives at the receiver having been scattered or reflected from irregularities located in the poleward wall of the trough or the auroral oval. This type of propagation occurs more often at sunspot maximum than at sunspot minimum [Warrington et al., 2007].

[29] 3. The delay spreads are generally higher at sunspot maximum than sunspot minimum. Higher-order modes tend to be more common at sunspot maximum, and this leads to the increase in the delay spreads. The various delay spreads (CMPS, EMPS, and IMPS) tend to be well correlated, but have different values. For example, CMPS and IMPS tend to be similar in value (within $0.5 \mathrm{~ms}$ of each other for between 90 and $95 \%$ of the time, depending on path and frequency), while they both tend to be higher than EMPS except for single-moded propagation where all three delay spreads are similar. Angling and Davies [1999] found that EMPS gave better results than CMPS when testing single tone modems using an HF channel simulator. However, IMPS has the benefit of being easy to determine both experimentally and using prediction programs such as VOACAP.

[30] 4. The agreement between predicted and measured values of IMPS is generally good at sunspot minimum, but poor at sunspot maximum. The poor agreement tends to arise because the effect of high-order modes (e.g., 3F2) is not well predicted.

[31] 5. The path length makes no difference to the Doppler spread and tends to result in little difference in the various delay spreads. However, the delay spreads are higher on the shorter path for lower frequencies during the day and for summer nights, while the opposite is the case for higher frequencies during equinoxes.

[32] The increased spreads (both delay and Doppler) during sunspot maximum will tend to have a deleterious effect on communications systems operating on paths in the subauroral region. For example, for DRM systems the nighttime Doppler spreads at sunspot maximum exceed the tolerance of a typical high data rate configuration (e.g., robustness mode B, maximum Doppler spread of $3 \mathrm{~Hz}$ [see Smith and Angling, 2003]) and also, at times, the lower data rate mode (robustness mode D, maximum Doppler spread of $6 \mathrm{~Hz}$ ). During these intervals, the system is likely to provide an unacceptable level of performance. While the multipath spreads observed on these paths are all within the operating range of the higher data rate $(21 \mathrm{kbit} / \mathrm{s})$ DRM modem (maximum delay spread is equal to $5 \mathrm{~ms}$ ), it is interesting to note that for lower frequencies on shorter paths, the higher values at sunspot maximum would probably exceed the maximum values and, thereby, lead to a reduction in data rate. Similar problems would occur with the systems using modems conforming to the STANAG 4285 standard. For example, such a system operating at a data rate of $2.4 \mathrm{kbit} / \mathrm{s}$ would fail if the Doppler spread were greater than $4.0 \mathrm{~Hz}$, a value that is exceeded during winter and equinoctial nights at sunspot maximum. As the next sunspot maximum approaches, it is clear that, at times, $\mathrm{HF}$ digital communications systems operating at subauroral latitudes will be strongly affected.

\section{References}

Angling, M. J., and N. C. Davies (1999), An assessment of a new ionospheric channel model driven by measurements of multipath and Doppler spread, paper presented at the Colloquium on Frequency Selection and Management Techniques for HF Communications, Inst. of Electr. Eng., London, 29-30 March.

Angling, M. J., P. S. Cannon, N. C. Davies, T. J. Willink, V. Jodalen, and B. Lundborg (1998), Measurements of Doppler and multipath spread on oblique high latitude HF paths and their use in characterizing data modem performance, Radio Sci., 33(1), 97-107, doi:10.1029/97RS02206.

Barclay, L., C. Behm, and S. Carroll (2009), Digitally modulated HF communications reliability: Modifications to ITU-R Rec. P.533 propagation model and the associated computer program REC533, IET Conf. Publ., 549, 69-73.

Davies, N. C., and P. S. Cannon (1993), DAMSON—A system to measure multipath dispersion, Doppler spread and Doppler shift on a multi-mechanism communications channels, paper presented at the Electromagnetic Wave Propagation Panel Symposium on Multiple Mechanism Propagation Paths: Their Characterisation and Influence on System Design, Advis. Group for Aerosp. Res. and Dev., Rotterdam, Netherlands, Oct.

Halcrow, B. W., and J. S. Nisbet (1977), A model of the F2 peak electron densities in the main trough region of the ionosphere, Radio Sci., 12, 815-820, doi:10.1029/ RS012i005p00815. 
International Telecommunication Union Radiocommunication Sector (ITU-R) (2000), Testing of HF modems with bandwidths of up to about $12 \mathrm{kHz}$ using ionospheric channel simulators, Recomm. ITU-R F.1487, Geneva.

International Telecommunication Union Radiocommunication Sector (ITU-R) (2007), Computation of reliability and compatibility of HF radio systems, Recomm. ITU $-R$ P.842-4, Geneva.

International Telecommunication Union Radiocommunication Sector (ITU-R) (2009), Method for the prediction of the performance of HF circuits, Recomm. ITU-R P.533-10, Geneva.

Lane, G. (2001), Signal-to-noise predictions using VOACAPA user's guide, Rockwell Collins Inc., Cedar Rapids, Iowa.

Rodger, A. S., R. J. Moffett, and S. Quegan (1992), The role of ion drift in the formation of ionization troughs in the midand high latitude ionosphere-A review, J. Atmos. Terr. Phys., 54(1), 1-30, doi:10.1016/0021-9169(92)90082-V.

Rogers, N. C. (2003), Optimising the placement of digital HF quality of service test receivers, and techniques for measurement extrapolation, in Proceedings of Ninth International Conference on HF Radio Systems and Techniques, IEE Conf. Publ., 493, 228-233.

Siddle, D. R., A. J. Stocker, and E. M. Warrington (2004a), Time-of-flight and direction of arrival of HF radio signals received over a path along the midlatitude trough: Observations, Radio Sci., 39, RS4008, doi:10.1029/2004RS003049.

Siddle, D. R., N. Y. Zaalov, A. J. Stocker, and E. M. Warrington (2004b), Time of flight and direction of arrival of HF radio signals received over a path along the midlatitude trough: Theoretical considerations, Radio Sci., 39, RS4009, doi:10.1029/2004RS003052.

Smith, O. J., and M. J. Angling (2003), Optimising quality of service of a new digital radio broadcasting system, in Proceedings of Ninth International Conference on HF Radio Systems and Techniques, IEE Conf. Publ., 493, 96-101.

Stocker, A. J., E. M. Warrington, and D. R. Siddle (2007), Comparison between the measured and predicted parameters of HF radio signals propagating along the midlatitude trough and within the polar cap, Radio Sci., 42, RS3019, doi:10.1029/2006RS003557.

Stocker, A. J., N. Y. Zaalov, E. M. Warrington, and D. R. Siddle (2009), Observations of HF propagation on a path aligned along the mid-latitude trough, Adv. Space Res., 44(6), doi:10.1016/j.asr.2008.09.038.

Warrington, E. M., and A. J. Stocker (2003), Measurements of the Doppler and multipath spread of HF signals received over a path oriented along the midlatitude trough, Radio Sci., 38(5), 1080, doi:10.1029/2002RS002815.

Warrington, E. M., A. J. Stocker, N. Y. Zaalov, and D. R. Siddle (2007), Observations of HF propagation on a path aligned along the mid-latitude trough, paper presented at Nordic HF Conference, Nordic Radio Soc., Fårö, Sweden.

Zaalov, N. Y., E. M. Warrington, and A. J. Stocker (2005), A ray-tracing model to account for off-great circle HF propagation over northerly paths, Radio Sci., 40, RS4006, doi:10.1029/2004RS003183.

Zaalov, N. Y., E. M. Warrington, and A. J. Stocker (2007), Effect of geomagnetic activity on the channel scattering functions of HF signals propagating in the region of the midlatitude trough and the auroral zone, Radio Sci., 42, RS4005, doi:10.1029/2006RS003611.

A. J. Stocker and E. M. Warrington, Department of Engineering, University of Leicester, Leicester LE1 7RH, UK. (emw@leicester.ac.uk) 